\title{
Proposta e Aplicação de Atividades para o Desenvolvimento das Habilidades de Organização de Informação e Pensamento Algorítmico
}

\author{
Placida Giuliane S. Oliveira ${ }^{1}$, Gean Carlos Brandao ${ }^{1}$, Luciana Foss ${ }^{1}$, André Du \\ Bois ${ }^{1}$, Marilton Aguiar', Renata Reiser' ${ }^{1}$, Clause Piana ${ }^{1}$, Ana Rita Mazzini ${ }^{1}$ \\ ${ }^{1}$ Centro de Desenvolvimento Tecnológico - Universidade Federal de Pelotas (UFPel) \\ Rua Gomes Carneiro, 1 - 96.010-610 - Pelotas - RS - Brasil \\ \{pgsdoliveira, gcbrandao, lfoss, dubois, marilton, \\ reiser\}@inf.ufpel.edu.br, clausepiana@yahoo.com.br, \\ anarita.mazzini@gmail.com
}

\begin{abstract}
With technology increasingly present in our daily lives, there is a change in the scenario of the labor market, which requires professionals more prepared to deal with problems. Computational thinking, a methodology that is based on the fundamentals of computer science for problem solving, is an innovation to increase and encourage improvements in teaching and learning and, therefore, in the preparation of these future professionals. This article aims to report two methodologies that aim to develop computational thinking skills in basic education through the process of unplugged activities.
\end{abstract}

Resumo. Com a tecnologia cada dia mais presente em nosso cotidiano, nota-se uma mudança no cenário do mercado de trabalho, que exige profissionais mais preparados para lidar com problemas. $O$ pensamento computacional, metodologia que se baseia nos fundamentos da ciência da computação para resolução de problemas, consiste numa inovação para incremento e incentivo de melhorias do ensino-aprendizagem e, portanto, no preparo destes futuros profissionais. Este artigo tem como objetivo o relato de duas metodologias que visam desenvolver habilidades do pensamento computacional no ensino básico através do processo de atividades desplugadas.

\section{Introdução}

À medida que a tecnologia avança, o mercado de trabalho muda suas necessidades, requerendo profissionais criativos, aptos a trabalhar em equipe e capaz de solucionar problemas interdisciplinares. Concomitante a essa realidade, populariza-se o pensamento computacional, uma metodologia que se baseia na aplicação dos fundamentos da ciência da computação para a resolução de problemas das mais diversas áreas do conhecimento.

Segundo [Wing 2006], pensamento computacional envolve a resolução de problemas, tanto computacionais quanto cotidianos, através dos conceitos fundamentais da ciência da computação. A autora defende que esta habilidade é fundamental para todos, não só para cientistas da computação, pois envolve a reformulação de um problema difícil em um problema que saibamos resolver. A importância do pensamento computacional bem como o estado da arte sobre a inclusão desta metodologia no Ensino Fundamental e Médio no Brasil vêm sendo estudado e apresentado [Bordini et al. 2017]. 
Este artigo apresenta uma proposta e relatos de aplicação de duas metodologias que visam promover habilidades do pensamento computacional, trabalhando atividades que envolvem os conceitos de classificação, seriação, análise, organização de dados e sequência lógica para alunos do $3^{\circ}$ ano do Ensino Fundamental. Além disso, as atividades também visam estimular o interesse dos alunos em leitura e escrita, incentivar o trabalho em equipe e favorecer o aprendizado sobre formas geométricas e interpretação de textos. O trabalho foi desenvolvido no escopo do projeto ExpPC ${ }^{1}$, na Universidade Federal de Pelotas (UFPel), que visa promover o pensamento computacional no âmbito do Ensino Fundamental. Há muitas propostas e relatos [Rodriguez et al. 2015, Guarda et al. 2018, Reis et al. 2017, Andrade et al. 2013 e Santana et al. 2017] que, assim como os presentes neste artigo, tem como objetivo desenvolver o pensamento computacional no Ensino Básico. Em particular, aqui neste trabalho foca-se na proposta de atividades que promovam o desenvolvimento das habilidades de classificação e pensamento algorítmico (sequência lógica) para alunos dos anos iniciais do Ensino Fundamental.

Devido à infraestrutura das escolas públicas do município de Pelotas, todas as tarefas foram elaboradas com base na metodologia da Computação Desplugada [Bell et al. 2011], que possibilita o desenvolvimento das habilidades do pensamento computacional de forma lúdica, mas sem o uso de computadores. Esta metodologia já foi aplicada em trabalhos como [Ferreira et al. 2015] que obteve resultados positivos. Neste contexto, o processo de ensino-aprendizagem é proposto do concreto ao abstrato, ou seja, numa primeira etapa são disponibilizados materiais lúdicos, de forma que elas possam compreender os conceitos manipulando objetos e interagir utilizando seus sentidos, para posteriormente trabalhar as relações que estabelecem tais conceitos.

O artigo está organizado como segue. A Seção 2 apresenta a proposta de atividade Classificação. A Seção 3 descreve brevemente a atividade Lógica dos Quadrinhos e traz um relato da aplicação das atividades propostas. Na Seção 4, os resultados da aplicação são apresentados e discutidos. A Seção 5 contém as considerações finais.

\section{Proposta de Atividade: Classificação}

Nesta seção, descreve-se uma atividade que envolve a construção de desenhos compostos de formas geométricas, como meio de abordar os fundamentos da classificação de objetos. A atividade é dividida em 5 tarefas, sendo a última um teste avaliativo, cada uma prevista para ocorrer num período de 1 hora. Nas primeiras quatro tarefas, os alunos são separados em grupos e recebem um kit de material composto de um conjunto variado de peças geométricas (com diferentes formatos e cores), uma folha de referência com o nome de cada figura geométrica e uma folha com o gabarito de uma figura a ser construída, conforme pode ser observado na Figura 1.

\footnotetext{
${ }^{1}$ Explorando o Pensamento Computacional para a Qualificação do Ensino Fundamental
} 

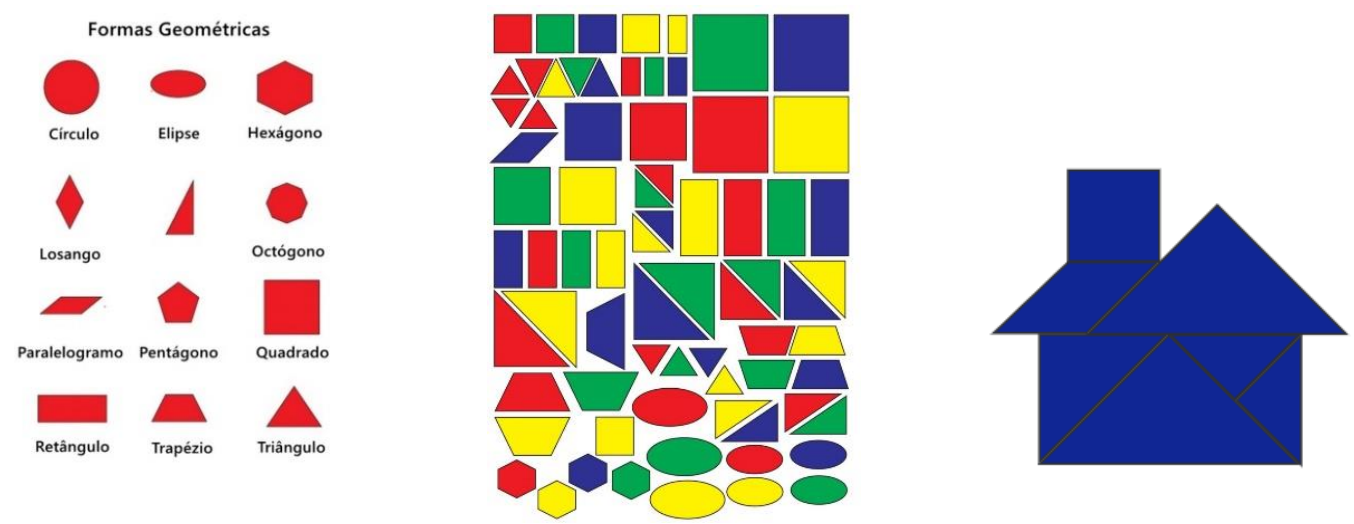

Figura 1. Folha de referência (à esquerda), parte do conjunto de peças (no centro) e exemplo de gabarito (à direita). Fonte: [ExpPC, 2018]

A partir deste kit, os estudantes constroem os desenhos sobrepondo as peças sobre o gabarito com o desenho impresso. Este gabarito contém as dimensões corretas do desenho, bem como a quantidade de peças e suas respectivas cores. Cada desenho foi elaborado com o objetivo de se utilizar pelo menos um critério de classificação para a organização das peças antes de sua montagem.

Para cada tarefa também foi proposto um método para avaliação, baseado em observações. O objetivo é de fazer uma análise mais precisa, considerando o desempenho em cada tarefa. Na sequência são detalhados os objetivos, metodologia e proposta de avaliação de cada tarefa. Todas as tarefas consideram uso de desenhos usando formas geométricas de diferentes formas e cores.

\subsection{Tarefa I: compreensão da classificação usando formas geométricas}

Objetivos: esta tarefa objetiva estimular os alunos a perceberem a importância da classificação por cor na construção de figuras geométricas.

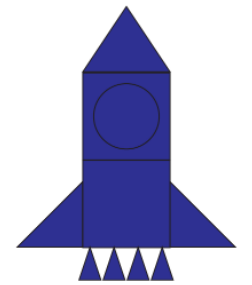

(a)

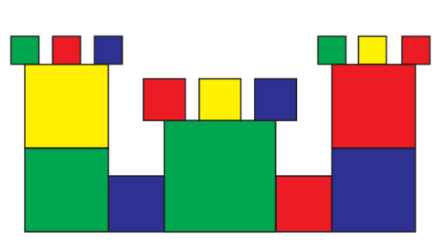

(b)

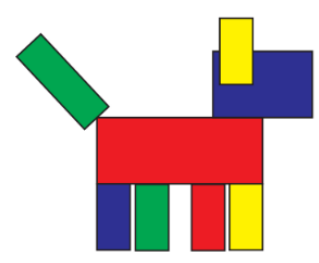

(c)

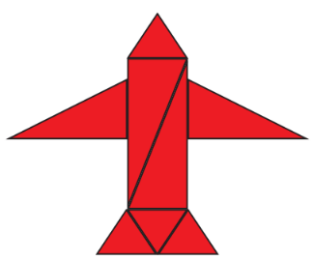

(d)

Figura 2. Figuras montadas na atividade de classificação. Fonte: [ExpPC, 2018]

Metodologia: após realizar uma introdução sobre o que são formas geométricas e como podemos usá-las para construir figuras mais complexas, os alunos são separados em grupos e recebem o kit de material, o qual deve ser utilizado para montar duas figuras propostas pelo professor. Cada desenho possui uma única cor e formas variadas, como no exemplo mostrado na Figura 2a.

A tarefa é dividida em duas etapas: na primeira etapa, os alunos devem montar as duas figuras utilizando as peças como receberam: desorganizadas. Na segunda etapa, 
VIII Congresso Brasileiro de Informática na Educação (CBIE 2019)

Anais do XXV Workshop de Informática na Escola (WIE 2019)

devem montar as mesmas figuras, porém, com as peças previamente separadas por cor. O tempo para a montagem de cada figura deverá ser cronometrado e anotado.

Ao final da aula, o professor pergunta aos alunos qual das duas maneiras facilitou o processo de montagem: peças separadas por cor ou todas misturadas. Ele explica que o objetivo da atividade foi fazê-los perceber a diferença de tempo e dificuldade de montar as figuras com ou sem um critério de classificação.

Avaliação: deve ser realizada por meio da observação do processo de montagem de cada figura: os grupos devem fazer a classificação adequada do material e chegar no objetivo que é a montagem correta das imagens propostas.

\subsection{Tarefa II: introdução do conceito de critérios de classificação}

Objetivos: esta tarefa tem como objetivo mostrar aos alunos que objetos podem ser classificados segundo diferentes critérios.

Metodologia: o professor inicia a aula fazendo uma breve revisão da aula anterior. A seguir, o kit de material é entregue aos alunos e é proposta a tarefa de separarem o conjunto de peças por formato. Depois de classificadas, o professor pede para que os grupos montem duas figuras: ambas de cores variadas e formadas apenas por peças com a mesma figura geométrica, como no exemplo da Figura $2 b$. Posteriormente, outras duas figuras são distribuídas: ambas compostas de somente uma cor e com formas variadas. Novamente, sob orientação do professor, devem realizar a classificação por cor e na sequência, montar a figura proposta.

Ao fim da tarefa propõe-se a discussão da necessidade de separar as figuras duas vezes e questiona-se por qual motivo a cada classificação foi utilizado um critério diferente. A discussão é conduzida para que os alunos compreendam a necessidade da definição de um critério que antecede a realização de uma classificação.

Avaliação: para avaliar o aprendizado desta tarefa, são solicitadas respostas a três perguntas, que devem ser feitas para cada grupo ao término da montagem, a respeito de cada figura montada. São estas: Utilizaram a mesma forma (e se não, quais formas usaram)? Usaram a mesma cor? Montaram a figura com todas as peças de mesmo tamanho?

\subsection{Tarefa III: definição e aplicação de critério para classificação}

Objetivos: esta tarefa tem como objetivo fazer com que os alunos definam e apliquem um critério de classificação descobrindo o que as peças da figura têm em comum.

Metodologia: o professor inicia a tarefa relembrando a aula anterior e em seguida entrega o kit de material aos grupos juntamente com três figuras a serem montadas: uma formada por retângulos de diferentes cores (como a Figura 2c), outra de cor azul com variados formatos e uma figura de mesma cor composta de apenas uma forma geométrica. Os alunos devem analisar as características em comum nas figuras para que, a partir disto, identifiquem o critério mais adequado para a classificação das peças. A análise da figura pode ser feita peça por peça, de forma a perceber o que as peças componentes possuem em comum.

Os alunos devem inicialmente definir o critério de classificação, para então organizar o material e por fim montar as figuras. Este processo deve ser repetido para cada figura a ser montada. 
VIII Congresso Brasileiro de Informática na Educação (CBIE 2019)

Anais do XXV Workshop de Informática na Escola (WIE 2019)

Ao final da aula, durante o espaço reservado para discussão, o professor deve explicar o motivo dos alunos terem de analisar cada figura e definir um critério antes de montá-las. Deve ser dito que nem todo o critério de classificação irá facilitar determinada tarefa. Por isto, devem ser analisadas as características comuns dos objetos antes de classificá-los.

Avaliação: para cada figura montada, foram feitas duas perguntas aos grupos para avaliar a compreensão da tarefa: O que há em comum na figura? É mais fácil classificar por cor ou forma (e por quê)?

\subsection{Tarefa IV: análise baseada na identificação de critérios de classificação}

Objetivos: esta tarefa tem como objetivo mostrar aos alunos que uma classificação pode ser feita baseada em mais de um critério.

Metodologia: nesta tarefa, após receberem o kit de material, os alunos analisam as duas figuras apresentadas pelo professor, ambas contendo apenas uma cor e uma forma, buscando um critério de classificação (exemplo na Figura 2d). Pede-se que, antes de partirem para a montagem da figura, os alunos classifiquem as peças nos critérios encontrados. Nas figuras distribuídas, é possível encontrar dois critérios (cor e forma) para classificar as figuras. E assim, os alunos são orientados a classificar o conjunto de peças segundo um dos critérios identificados e então, para o subgrupo de peças que possui uma das características de interesse, solicita-se que seja classificado novamente considerando o outro critério.

Ao final da aula, o professor reforça a importância de analisar a figura antes de realizar uma classificação. Também, deve explicar que utilizar mais de um critério de classificação pode facilitar ainda mais a montagem correta das figuras.

Avaliação: foram propostas duas perguntas aos grupos, para cada figura montada: Qual foi o primeiro critério utilizado para organizar as peças, para montar a figura? Qual outro critério podemos utilizar?

\subsection{Tarefa V: método de avaliação}

Objetivos: esta tarefa busca aplicar um conjunto de questões relativo ao que foi visto ao longo da atividade Classificação, onde serão avaliados os conhecimentos adquiridos.

Metodologia: uma avaliação contendo todos os conceitos trabalhados nas aulas anteriores deve ser aplicada aos alunos, levando em conta os critérios de classificação considerados na montagem das figuras.

\section{Relato da Aplicação}

A atividade descrita na Seção 2 foi aplicada a uma turma de terceiro ano do Ensino Fundamental do município de Pelotas. Juntamente com essa atividade, foi também aplicada a atividade "Lógica dos Quadrinhos" proposta em [Martin et al. 2017]. A mesma metodologia foi considerada nesta atividade, divididas em tarefas e aplicadas em encontros semanais de 1 hora de duração cada.

Na sequência, na Subseção 3.1 tem-se um breve relato da aplicação da atividade “Classificação". Na Subseção 3.2 a descrição da atividade "Lógica dos Quadrinhos" e 
VIII Congresso Brasileiro de Informática na Educação (CBIE 2019)

Anais do XXV Workshop de Informática na Escola (WIE 2019)

o relato de sua aplicação foram apresentados. As atividades completas, com os planos de aula e materiais, estão disponíveis no site $^{2}$ do projeto.

\subsection{Receptividade da Atividade Classificação}

A turma inicialmente recebeu a atividade com curiosidade devido às variadas cores $\mathrm{e}$ formas das figuras. Notou-se dificuldade no reconhecimento das formas geométricas, o que foi contornado com uma breve introdução sobre o assunto, incluindo a folha de referência (Figura 1) ao kit de material das aulas. O grupo teve desempenho satisfatório em reconhecer os padrões, classificar as figuras e ainda mais relevante, em atividades lúdicas de trabalho em equipe.

\subsection{Descrição Metodológica da Atividade Lógica dos Quadrinhos}

Esta atividade propõe trabalhar com estórias em quadrinhos visando ensinar sequência lógica. A atividade propõe cinco tarefas, incluindo uma avaliação para mensurar o aprendizado das tarefas via metodologia desenvolvida. As tarefas aplicadas estão brevemente descritas logo a seguir.

\section{Tarefa I: introdução da metodologia de estórias em quadrinhos}

Nesta tarefa introdutória, descreveu-se para a turma o método de contar histórias em quadrinhos. A turma foi dividida em duplas, recebeu o material contendo cinco conjuntos de tirinhas recortadas e embaralhadas. Solicitou-se que os alunos as organizassem de modo que cada sequência apresentada fizesse sentido (narrando uma estória). Um exemplo é ilustrado na Figura 3.
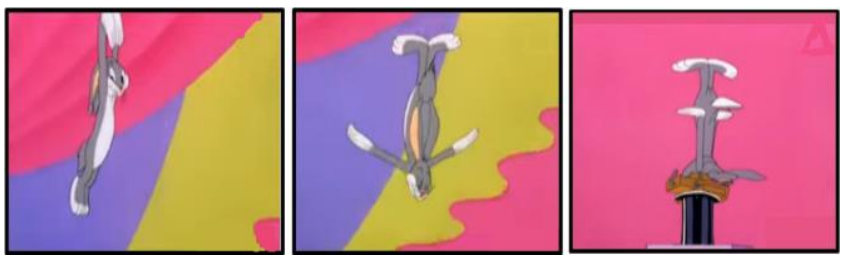

Figura 3. Exemplo de quadrinhos. Fonte: [BROS 1942]

\section{Tarefa II: introduzindo a consistência lógica das estórias em quadrinhos}

Foram distribuídos um quadrinho com gravura e um quadrinho em branco (conforme pode ser visto na Figura 4a. Os alunos, individualmente, completaram o quadrinho em branco com um desenho que fizesse sentido em relação ao desenho anterior, dando sequência lógica à história.

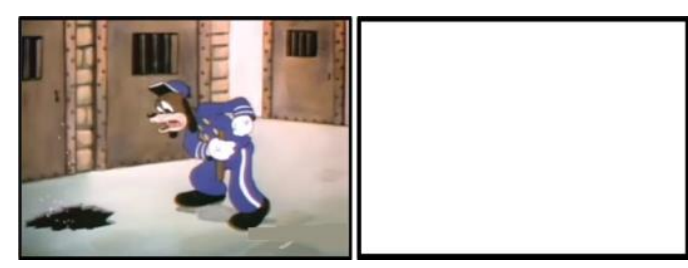

(a)

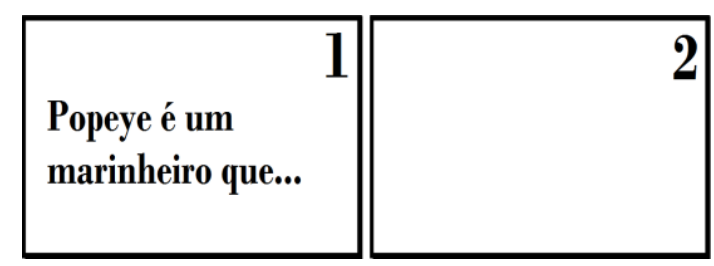

(b)

Figura 4. Exemplo de quadrinhos utilizados nas tarefas. Fonte: [BROS 1939]

\footnotetext{
${ }^{2}$ https://wp.ufpel.edu.br/pensamentocomputacional/pt
} 
VIII Congresso Brasileiro de Informática na Educação (CBIE 2019)

Anais do XXV Workshop de Informática na Escola (WIE 2019)

\section{Tarefa III: enfatizando a sequencialidade das estórias em quadrinhos}

A tarefa 3 foi realizada em grupos de três alunos, para cada grupo foi entregue um quadrinho enumerado, contendo uma frase que daria início a uma história, e um quadrinho em branco enumerado com a sequência do anterior para ser utilizado para dar seguimento a esta história. Os alunos poderiam optar por escrever ou desenhar a continuação (conforme a Figura 4b). Feito isto, o grupo passou esta história para outro grupo, entregando apenas o último quadrinho. Os alunos continuaram a escrever nas tirinhas até que todas as histórias passassem por todos os grupos.

\section{Tarefa IV: estimulando a criatividade nas estórias em quadrinhos}

Nesta tarefa, os alunos criaram suas próprias histórias com, no mínimo, três quadrinhos. A seguir, elas foram entregues a outros alunos e foi pedido para que eles as organizassem em sequência, assim como na tarefa 1.

\section{Tarefa V: método de avaliação}

$\mathrm{Na}$ avaliação, foi entregue aos alunos um conjunto de questões que abordavam as tarefas anteriores, com o objetivo de medir a aprendizagem e desempenho das tarefas de avaliação propostas na turma.

A proposta de atividade descrita em [Martin et al. 2017], apresenta uma tarefa 5, que foi considerada como avaliação das demais tarefas. Além disso, em cada etapa foi pedido para que os alunos escrevessem uma breve frase justificando cada quadrinho organizado por eles. Garantindo assim, uma maneira de avaliar cada aula individualmente, através das justificativas dadas pelos alunos.

Percebeu-se ainda que a turma tinha alguns alunos que não sabiam ler, estes foram ajudados pelos colegas e aplicadores com as atividades. Também observou-se que os alunos tinham facilidade em organizar as figuras e justificá-las nas sequências e organização lógica.

\section{Resultados e discussão}

Os alunos receberam uma nota por seu desempenho, numa escala de 0 a 10, em cada uma das tarefas realizadas em cada atividade. Na última tarefa de cada atividade, foram submetidos a uma avaliação envolvendo as habilidades desenvolvidas no conjunto das tarefas.

$\mathrm{Na}$ atividade Lógica dos Quadrinhos, foram realizadas as seguintes tarefas: (1) encontrar a sequência correta das cenas de uma história em quadrinhos; (2) a partir de uma cena fornecida, desenhar a próxima cena de uma história em quadrinhos; (3) criar uma história em grupo, onde cada aluno desenha uma cena que dará sequência à cena anterior; (4) desenhar uma história completa. A atividade Classificação também compreendeu quatro tarefas: (1) classificação de objetos por cor; (2) classificação de objetos por cor e forma; (3) definição de critérios para classificação; e (4) definição de múltiplos critérios de classificação.

A descrição do desempenho dos alunos por Tarefa e na avaliação final de cada atividade é apresentada na Tabela 1 . Note-se que o número de alunos variou entre as Tarefas e as avaliações em razão de eventuais ausências nas aulas. 
VIII Congresso Brasileiro de Informática na Educação (CBIE 2019)

Anais do XXV Workshop de Informática na Escola (WIE 2019)

Tabela 1. Descrição do desempenho dos alunos nas tarefas e na avaliação final, por atividade.

\begin{tabular}{l|c|cccc|ccccc}
\hline & Tarefa & $\mathbf{n}$ & Média & $\mathbf{D P}$ & $\begin{array}{c}\mathbf{C V} \\
\mathbf{( \% )}\end{array}$ & Mínimo & $\mathbf{Q}_{\mathbf{1}}$ & $\mathbf{M d}$ & $\mathbf{Q}_{\mathbf{3}}$ & Máximo \\
\hline \multirow{4}{*}{$\begin{array}{l}\text { Lógica dos } \\
\text { Quadrinhos }\end{array}$} & $\mathbf{1}$ & 14 & 8,0 & 1,0 & 12,5 & 7 & 7,2 & 7,6 & 8,6 & 10 \\
& $\mathbf{3}$ & 14 & 7,2 & 2,6 & 36,5 & 2 & 5 & 8,7 & 8,7 & 10 \\
& $\mathbf{4}$ & 17 & 10,0 & 0 & 0 & 10 & 10 & 10 & 10 & 10 \\
\cline { 2 - 10 } & Avaliação & $\mathbf{1 7}$ & $\mathbf{7 , 5}$ & $\mathbf{1 , 1}$ & $\mathbf{1 4 , 1}$ & $\mathbf{5 , 6}$ & $\mathbf{6 , 6}$ & $\mathbf{7 , 8}$ & $\mathbf{8 , 2}$ & $\mathbf{9 , 2}$ \\
\hline \multirow{5}{*}{ Classificação } & $\mathbf{1}$ & 13 & 7,7 & 4,4 & 57,0 & 0 & 10 & 10 & 10 & 10 \\
& $\mathbf{2}$ & 14 & 7,3 & 3,3 & 45,6 & 0 & 5,3 & 9,3 & 9,5 & 10 \\
& $\mathbf{3}$ & 14 & 6,6 & 3,1 & 46,6 & 3,3 & 3,3 & 7,5 & 9,2 & 10 \\
& $\mathbf{4}$ & 12 & 7,5 & 3,2 & 43,2 & 1,3 & 5 & 10 & 10 & 10 \\
\cline { 2 - 10 } & Avaliação & $\mathbf{1 3}$ & $\mathbf{6 , 5}$ & $\mathbf{2 , 5}$ & $\mathbf{3 8 , 7}$ & $\mathbf{0}$ & $\mathbf{6}$ & $\mathbf{7}$ & $\mathbf{8}$ & $\mathbf{1 0}$ \\
\hline
\end{tabular}

Nota: $\mathrm{n}=$ número de alunos; $\mathrm{DP}=$ desvio padrão; $\mathrm{CV}=$ Coeficiente de variação; $\mathrm{Q}_{1}=$ primeiro quartil; $\mathrm{Md}$ $=$ mediana; $\mathrm{Q}_{3}=$ terceiro quartil.

Como pode ser observado, o desempenho geral da turma na atividade Lógica dos Quadrinhos foi satisfatório. A média ficou próxima de 7,0 nas Tarefas 2 e 4 e foi mais elevada nas Tarefas 1 e 3 (8,0 e 10,0, respectivamente). Verificou-se também que variação das notas foi alta $(\mathrm{CV}=36 \%)$ na Tarefa 2 e muito alta $(\mathrm{CV}=62 \%)$ na Tarefa 4 . Na avaliação final, que compreendia o conteúdo desenvolvido em todas as tarefas, as notas da turma variaram de 5,6 a 9,2, com média 7,5. Nesta avalição, pelo menos 50\% da turma alcançou nota maior ou igual a 7,8 .

$\mathrm{Na}$ atividade Classificação o desempenho geral da turma foi um pouco inferior ao da atividade Lógica dos Quadrinhos. Os resultados mostram que em todas as Tarefas a média da turma não se afastou muito de 7,0, mas a variabilidade das notas foi muito elevada, com CV variando entre $43 \%$ e $57 \%$. Na avaliação final, as notas da turma variaram de 0 a 10, com média 6,5. Embora 50\% da turma tenha alcançado nota maior ou igual a 7,0 nesta avaliação, $25 \%$ apresentaram nota entre 0 e 6.

Os alunos também foram submetidos a um teste envolvendo classificação e pensamento algorítmico, que foi aplicado em dois momentos: antes (pré-teste) e depois (pós-teste) do desenvolvimento das atividades. Este teste compreendeu dez questões (valendo 1,0 ponto cada) relacionadas às habilidades trabalhadas nas atividades. A finalidade deste teste foi avaliar se as atividades do projeto melhoram as habilidades dos alunos na resolução das questões

A pontuação no pré-teste descreve a condição inicial do aluno em relação às habilidades trabalhadas nas atividades. $\mathrm{O}$ ganho do aluno é mensurado pela diferença entre as notas obtidas no pós e no pré-teste. As medidas descritivas das notas no pré e no pós-teste e do ganho dos alunos é apresentada na Tabela 6. 
VIII Congresso Brasileiro de Informática na Educação (CBIE 2019)

Anais do XXV Workshop de Informática na Escola (WIE 2019)

Tabela 6. Medidas descritivas das notas e do ganho dos alunos no teste.

\begin{tabular}{c|cccc|ccc}
\hline Teste & $\begin{array}{c}\text { Número de } \\
\text { alunos }\end{array}$ & Média & $\begin{array}{c}\text { Desvio } \\
\text { padrão }\end{array}$ & $\begin{array}{c}\text { Coeficiente } \\
\text { de variação } \\
(\%)\end{array}$ & Mínimo & Mediana & Máximo \\
\hline Pré & 14 & 4,4 & 2,2 & 50,6 & 1 & 4 & 7 \\
Pós & 14 & 6,4 & 2,3 & 36,5 & 2 & 7,5 & 9 \\
\hline Ganho & 14 & 2,1 & 2,3 & - & -2 & 1,5 & 7 \\
\hline
\end{tabular}

Verifica-se que, em média, os alunos tiveram um ganho de 2,1 pontos no pósteste, indicando que houve melhora no desempenho geral da turma, mas o desvio padrão de 2,3 pontos revela que a variação foi elevada. A significância desse ganho foi verificada por meio de um teste $t$ para amostras pareadas, com $\alpha=0,05$. O teste evidenciou que $o$ ganho dos alunos foi altamente significativo $(p=0,0051)$.

\section{Considerações Finais}

O objetivo deste artigo foi relatar as atividades propostas e aplicadas no ano de 2018 no projeto de extensão ExpPC, para uma turma de $3^{\circ}$ ano do Ensino Fundamental. Através da análise dos resultados apresentada na Seção 4, percebeu-se que a turma obteve ganho em suas habilidades relacionadas ao pensamento computacional. Comprovando assim, que através da inclusão de atividades desplugadas relacionadas a esta habilidade, pode-se disseminar a metodologia do pensamento computacional nas escolas.

Salientam-se ainda relevantes a interação de professores e alunos do ensino fundamental com professores e alunos de graduação dos cursos de Computação. A preparação do material didático, planos de ensino e de atividades bem como a metodologia de apresentação, propiciaram experiência didática enriquecedora para todos agentes envolvidos, além do acompanhamento às atividades de extensão e divulgação junto a comunidade escolar e acadêmica.

E, sobretudo renovaram-se posturas, com incentivo à maior interação entre aluno/professor nas escolas de ensino fundamental bem como o intercâmbio de visões científicas e/ou extensionistas entre orientando e/ou orientador nos cursos acadêmicos da Computação da Universidade Federal de Pelotas (UFPel). Todos estes agentes foram e serão importantes para enfrentar o desafio de organização e desenvolvimento de novos métodos e tecnologias envolvendo atividades de ensino/aprendizagem baseadas no pensamento computacional.

Em trabalhos futuros, buscamos propor novas atividades lúdicas e desplugadas via metodologias baseadas no pensamento computacional, de forma a englobar todas as habilidades propostas nas Diretrizes Curriculares da Sociedade Brasileira de Computação para o $3^{\circ}$ ano do Ensino Fundamental.

\section{Referências}

Andrade, D. et al. (2013). Proposta de atividades para o desenvolvimento do pensamento computacional no ensino fundamental. In Anais do XIX WIE, p. 169-178.

Bell, T.; Witten, I, H.; Fellows, M. (2011). “Computer Science Unplugged - Ensinando Ciência da Computação sem o uso do computador”. Tradução coord. por Luciano 
VIII Congresso Brasileiro de Informática na Educação (CBIE 2019)

Anais do XXV Workshop de Informática na Escola (WIE 2019)

Porto Barreto, 2011. Disponível em <http://csunplugged.org/>. Acesso em junho/2019.

Bordini, Adriana; Avila, Christiano; Marques, Monica; Foss, Luciana; Cavalheiro, Simone. Pensamento Computacional nos Ensinos Fundamental e Médio: uma revisão sistemática. In XXVIII Simpósio Brasileiro de Informática na Educação SBIE (Brazilian Symposium on Computers in Education), 2017, Recife, 2017. p. 123-132.

Brackmann, Christian Puhlmann. Pensamento Computacional Brasil. 2019. Disponível em <http://www.computacional.com.br/> Acesso em junho/2019.

Bros., W. (1939). Bars and Stripes Forever. Disponivel em <http://looneytunes.wikia.com/wiki/Bars_and_Stripes_Forever>. Acesso em junho/2019. ADAPTADO.

Bros., W. (1942). Case of the Missing Hare. Disponível em <http://looneytunes.wikia.com/wiki/Case_of_the_Missing_Hare>. Acesso em junho/2019. ADAPTADO.

Explorando o Pensamento Computacional para a Qualificação do Ensino Fundamental ExpPC. Disponível em: <https://wp.ufpel.edu.br/pensamentocomputacional/pt $>$

Ferreira, A. C. C., Melhor, A., Barreto, J. S., Paiva, L. F., Matos, E. (2015). "Experiência Prática Interdisciplinar do Raciocínio Computacional em Atividades de Computação Desplugada na Educação Básica". In Anais do XXI WIE, p. 256-265.

Guarda, Graziela; Goulart, Ione. Jogos Lúdicos sob a ótica do Pensamento Computacional: Experiências do Projeto Logicamente. In XXIX Simpósio Brasileiro de Informática na Educação (Brazilian Symposium on Computers in Education), 2018, Fortaleza, 2018. p. 486.

Martin, S. S. ; Cavalheiro, S. A. C. ; Foss, L. ; Aguiar, M. S. ; Reiser, R. H. S. (2017) "Desenvolvendo o raciocínio lógico utilizando quadrinhos: uma proposta de atividade para o terceiro ano do ensino fundamental via pensamento computacional". In Anais do IV WEIT, p. 120-127.

Reis, F. M.; Oliveira, F. C. S., Martins, D. J. S., Moreira, P. R. (2017) "Pensamento Computacional: Uma Proposta de Ensino com Estratégias Diversificadas para Crianças do Ensino Fundamental". In Anais do XXIII WIE, p. 638-647.

Rodriguez, C. L.; Zem-Lopes, A. M., Marques, L. e Isotani, S. (2015) "Pensamento Computacional: transformando ideias em jogos digitais usando o Scratch". In Anais do XXI WIE, p. 62-71.

Santana, A. L. M; Jesus, E. A., Cucco, L. A., Santana, L. F. M., Raabe, A. L. A., Ramos, G. L. (2017) "Tem Ideia na Rede: Inserindo o Pensamento Computacional na Rede Municipal de Ensino". In Anais do XXIII WIE, p. 1032-1041.

Wing, J. PENSAMENTO COMPUTACIONAL - Um conjunto de atitudes e habilidades que todos, não só cientistas da computação, ficaram ansiosos para aprender e usar. Revista Brasileira de Ensino de Ciência e Tecnologia, v. 9, n. 2, 2016. 